\title{
Applications of Multimodal Physical (IoT), Cyber and Social Data for Reliable and Actionable Insights
}

\author{
Amit Sheth \\ Kno.e.sis Center, \\ Wright State University \\ Dayton, $\mathrm{OH}$, USA \\ amit@knoesis.org
}

\author{
Pramod Anantharam \\ Kno.e.sis Center, \\ Wright State University \\ Dayton, $\mathrm{OH}$, USA \\ pramod@knoesis.org
}

\author{
Krishnaprasad \\ Thirunarayan \\ Kno.e.sis Center, \\ Wright State University \\ Dayton, $\mathrm{OH}$, USA \\ tkprasad@knoesis.org
}

\begin{abstract}
Physical objects with embedded sensors are increasingly being networked together using wireless and internet technologies to form Internet of Things (IoT). However, early applications that rely on IoT data fail to provide comprehensive situational awareness. This often requires combining physical (i.e., IoT) data with social data created by humans on the Web and increasingly on their mobile phones (i.e., citizen sensing) as well as other data such as structured open data and background knowledge available on the Web (i.e., cyber data and knowledge). In this paper, we explore how integration and analysis of multimodal physical-cybersocial data can support advanced applications and enrich human experience. Specifically, we illustrate the complementary role played by sensor and social data, often intermediated by other Web based data and knowledge, using real-world examples in the domain of situational awareness, traffic monitoring, and healthcare. We also show how semantic techniques and technologies support critical data interoperability needs, advanced computation capabilities including reasoning, and significantly enhance our ability to exploit growing amount of data from the proliferation of Internet of Things.
\end{abstract}

\section{INTRODUCTION}

Internet of Things (IoT) has profound impact on interactions between people with their environment [6]. By 2020, it is estimated that there will be over 50 billion devices on the Internet $^{1}$. Analyzing sensor data from this massive network of interconnected devices poses formidable big data challenges due to the volume, velocity, variety and veracity issues, and our inability to extract usable value from them[10]. We hypothesize that multimodal data analytics obtained by

\footnotetext{
${ }^{1}$ http://internetofeverything.cisco.com/
}

combining textual observations from people (henceforth referred to as social data) and sensor data from IoT can go a long way in harnessing actionable insights.

In what follows, we illustrate the complementary role played by sensor, Web and social data (on social networking sites like twitter) using real-world examples involving situational awareness, traffic monitoring, and healthcare, to make the case that their integration and analysis will provide comprehensive and accurate understanding, and facilitate decision making. We also motivate the application of semantic techniques and technologies to facilitate realization and enrichment of IoT at physical, cyber, and social levels to assist and enhance all forms of human activities in a natural way.

\section{APPROACH AND APPLICATIONS}

We discuss the benefits of combining multimodal data to obtain reliable and actionable insights using three application scenarios.

\subsection{Situation Awareness}

In most real-world environments, there are many different types of sensors observing a situation. In a military context shown in Figure 1, the machine sensors can be as diverse as satellites, cameras, and GPS sensors, to weather station sensors providing temperature, wind speed, and precipitation measurements. Social sensors (a.k.a. citizen sensors) can provide situational information using smartphones (to deliver text messages via SMS) and via social networking sites (e.g., Twitter, Facebook).

Machine sensors are objective and provide quantitative observational values by measuring low-level phenomenon; they are persistent and precise to a degree that cannot be matched by a human observer. On the contrary, social sensors can be subjective and capable of providing a qualitative comprehensive interpretation of a situation very quickly and succinctly. Specifically, similar machine sensed values can have different real-world interpretations from the eye of an observer or a decision maker. Thus, machine sensors and social sensors can provide complementary and corroborative information. Ultimately, to provide decision makers with comprehensive and accurate situation awareness, reasoning will be essential to proactively seek relevant information, and integrate and abstract sensor data to obtain human comprehensible information, using semantic perception [7]. Figure 2 shows the application of multiple ontologies for describing different sensors and sensor observations, and for 
Figure 1: Integration of heterogeneous sensor data in military context

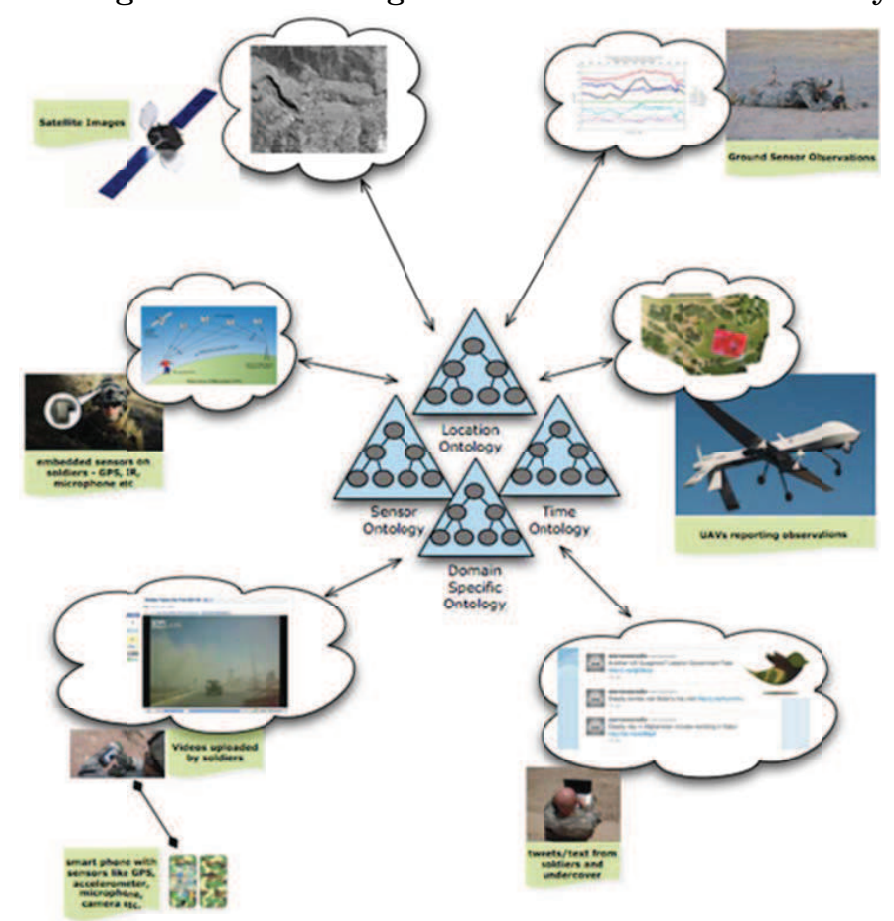

- http:I/mww.break.com/usercontent/2008/1/Soldier-Takes-His-First-IED-Hil-441384 - http://www.onlinesocialmedia.net/20100913/soldier-to-tweet-from-front-line-in-afghanistan

Figure 2: Use of multiple ontologies in different contexts and for different purposes

\begin{tabular}{|ccccc|c|}
\hline $\begin{array}{c}\text { Fixed machine } \\
\text { Sensor }\end{array}$ & $\begin{array}{c}\text { Sensor/Observation } \\
\text { Ontology }\end{array}$ & Spatial Ontology & Temporal Ontology & Domain Ontology \\
$\begin{array}{c}\text { Mobile machine } \\
\text { Sensor }\end{array}$ & Capabilities of sensor & $\begin{array}{c}\text { Location of } \\
\text { sensor }\end{array}$ & $\begin{array}{c}\text { Time when sensor is } \\
\text { active }\end{array}$ & $\begin{array}{c}\text { Type of qualities that } \\
\text { can be observed }\end{array}$ \\
$\begin{array}{c}\text { Fixed machine } \\
\text { observation }\end{array}$ & Detecting sensor & $\begin{array}{c}\text { Location of } \\
\text { observation }\end{array}$ & Time of observation & $\begin{array}{c}\text { Type of quality } \\
\text { active }\end{array}$ & $\begin{array}{c}\text { observed } \\
\text { can be observed }\end{array}$ \\
$\begin{array}{c}\text { Mobile machine } \\
\text { observation }\end{array}$ & Detecting sensor & $\begin{array}{c}\text { Location of } \\
\text { observation }\end{array}$ & Time of observation & $\begin{array}{c}\text { Type of quality } \\
\text { observed }\end{array}$ \\
$\begin{array}{c}\text { Human } \\
\text { observation }\end{array}$ & Detecting sensor & $\begin{array}{c}\text { Location of } \\
\text { observation }\end{array}$ & Time of observation & $\begin{array}{c}\text { Type of quality } \\
\text { observed }\end{array}$ \\
\hline
\end{tabular}

information along different dimensions, that will act as a foundation for semantic processing of heterogeneous data.

In order to better appreciate the benefits of integrating social and sensor data, we discuss their relative strengths and weaknesses from the perspective of situation awareness applications. Machine sensing is prone to vagaries of environmental effects and malicious attacks, while social sensing (a.k.a. citizen sensing) is prone to cognitive bias, rumors, misunderstandings, and factual inaccuracies, accidentally by uninformed public or intentionally by miscreants [2]. In order to improve trustworthiness of data provided by a sensor network, and to enable detection of anomaly, it is important to be able to cross check data provided by different sensors of a sensor network. For densely populated homogeneous sensor networks, one can expect and exploit spatio-temporal coherence in sensor data generated by sensors in spatio-temporal proximity. As such statistical methods may be brought to bear to ascertain their consistency [9]. On the other hand, such syntactic approaches are inadequate when dealing with heterogeneous sensor networks that contain sensors measuring different phenomenon (even when housed in the same sensor station), but where different sensor stations are relatively far apart. In such cases, domain models capturing semantic correlation among sensor data are necessary to reconcile semantic heterogeneity. (For example, the color of the flame of a burning object and its temperature are correlated; values from co-located magnetometers, accelerometers, gyroscopes, and GPS are correlated.) Furthermore, anomaly detection in thinly populated sensor networks can require background knowledge specifying normalcy as a function of spatio-temporal coordinates. This issue is further complicated by the fact that "unexpected" sensor data may in fact represent an abnormal situation. That is, it may be impossible to distinguish an abnormal situation from a sensor fault purely on the basis of observational data. In order to deal with this situation, we need to construct sensor fault models. Analogously, in the context of anomalous social data, we need to distinguish between occurrences of an abnormal event from utterances of uninformed public or malicious elements [2]. We need to develop robust cause-effect models taking into account normalcy, faults, context and intentions to distinguish data from compromised sensors (resp. malicious agent), legitimate data signaling abnormal situation (resp. unlikely 
Figure 3: A slow moving traffic reported by sensor observations on 511.org is supported by a sporting event that is scheduled in the sporting arena

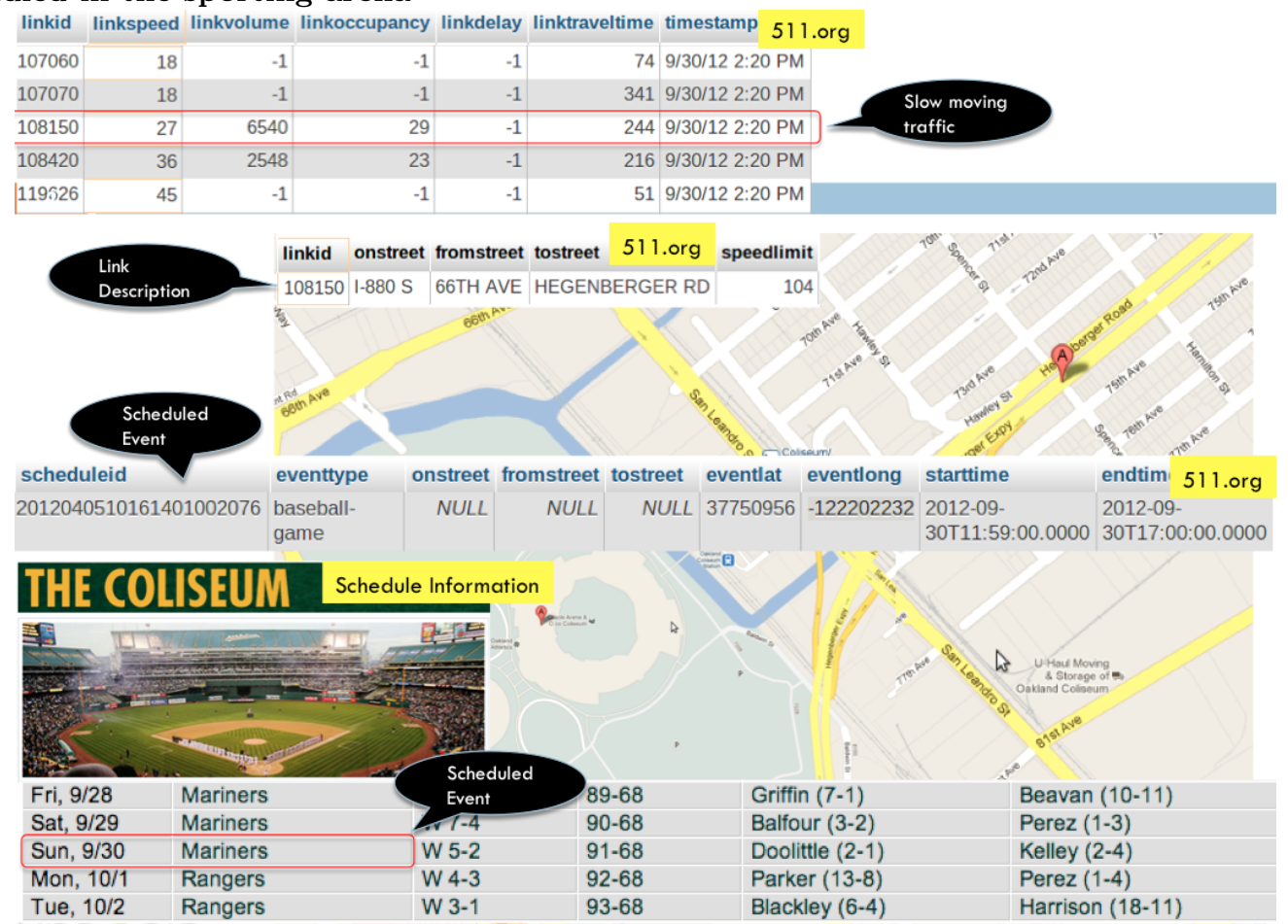

event), and erroneous data from faulty sensors (resp. uninformed public).

Wireless sensor network nodes normally offload their sensed data, periodically or triggered by an event, to a data sink that is continuously connected to the Internet. In contrast, unattended wireless sensor network nodes are relatively sporadically polled by a mobile data sink, requiring extra power, storage, and security measures to keep the data intact. The latter nodes, which are commonly deployed in areas such as battle fields and border crossings, are more prone to compromise, due to illegal data readouts and tampering. To reduce power requirements and to avoid promiscuous readouts, sensors and RFID technologies are being combined to facilitate passive communication using RFID readers power and to improve security with reads enabled only in an appropriately sensed semantic (spatio-temporal-thematic) context.

Qualitative observations from people are imprecise, unreliable, and subjective. Processing qualitative observations also require careful consideration for dealing with the challenges analogous to those mentioned above. In summary, multimodal data from sensors and social streams can improve reliability and comprehensiveness of situation awareness.

\subsection{Traffic}

Traffic is a major concern in many cities around the world and understanding traffic related events is an important problem for city authorities.

Sensors and devices connected on IoT networks constantly report observations of the physical world, ranging from the environment that surrounds us to traffic conditions on the roads that allow us to move around. Sensor observations may be in the form of stream of numbers (e.g., indicating temperature, humidity, and traffic flow rate) or booleans (e.g., indicating presence or absence of a car in a parking space). Observations from people, in contrast, are qualitative in nature and easily human comprehensible (e.g., messages indicating slow traffic or icy roads). Furthermore, multimodal data integration and abstraction can provide more comprehensive picture of the traffic conditions because they provide complementary view of the same situation. For example, a slow moving traffic registered by a traffic flow sensor can be explained by anything from rush hour traffic on a weekday, to that prevailing before and after a rock concert near its arena, to that caused by a car accident or due to icy road conditions. In general, the real-world event that explains the machine sensed observation can be determined systematically by checking if the prevailing situation can be accounted for by typical traffic pattern based on the spatiotemporal context, or by an occurrence of a planned event, or in exceptional but critical cases, by gleaning cause from socially sensed observations sent via tweets from mobile devices. Figure 4 provides an interesting example of utilizing textual streams with IoT as a complementary (that is, providing additional information or interpretation) and corroborative (that is, further confirming the event) source of information. Slow moving traffic (marked red) is reported by sensors on Buckeye Traffic ${ }^{2}$. However, the cause of slow moving traffic is not directly observed by machine sensors. We found that an accident, specifically, an overturned semitrailer, was reported on twitter. Such a reported observation provides additional information that can be beneficial in the context of IoT where ultimately people are looking

\footnotetext{
$\overline{{ }^{2} \text { http://www.buckeyetraffic.org/ }}$
} 
Figure 4: Sensor observations reporting slow traffic (marked red) and two other complementary sources of information (a) accident reported on twitter (b) accident reported on news channel

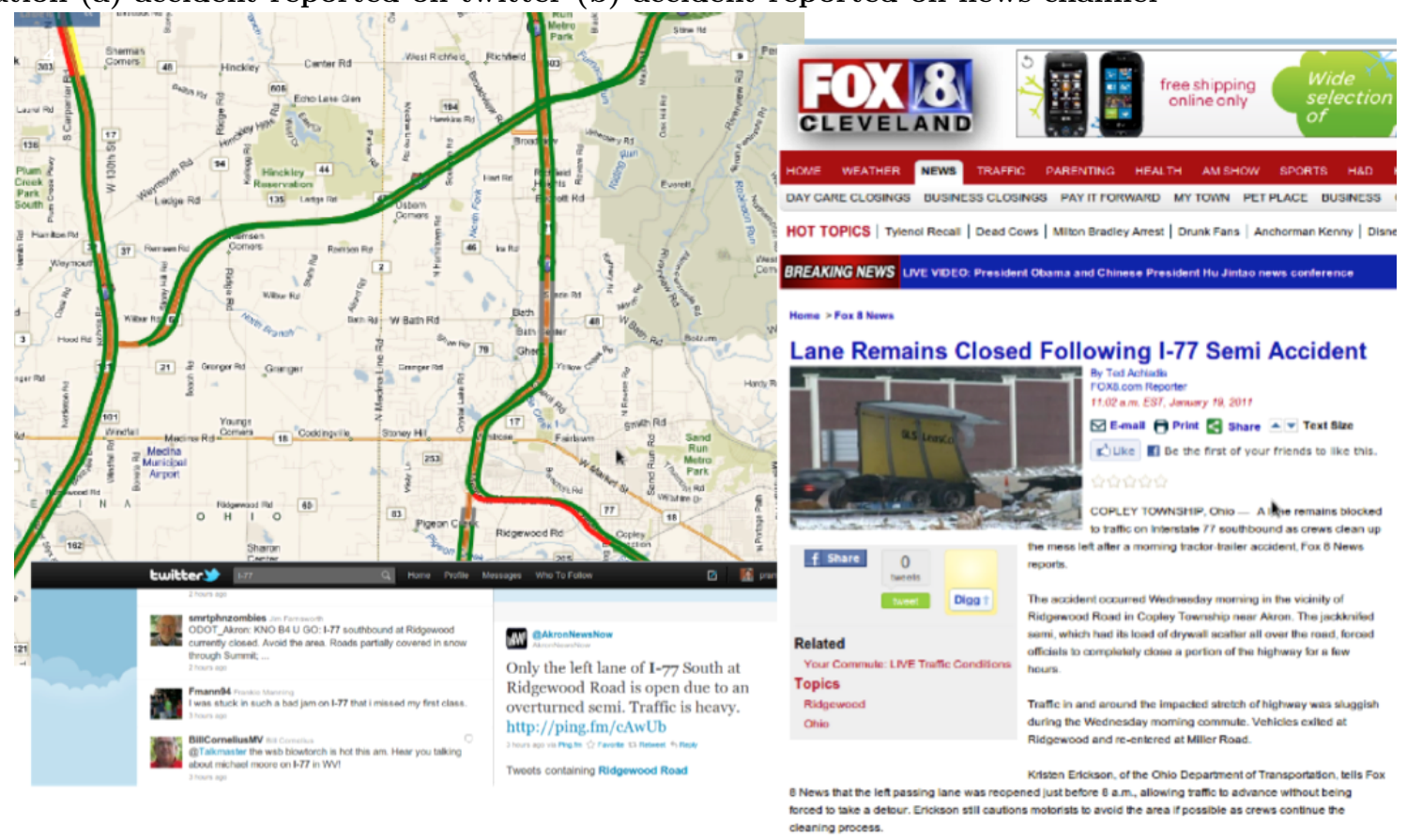

for comprehensive and human comprehensible insights for future action.

Social data such as twitter stream can be both noisy and diffuse. So we have developed effective techniques exploiting linear chain conditional random fields and geohashing to enable recognition and annotation of traffic events in tweets [1]. In order to evaluate the role and impact of social streams for deriving traffic events, we studied the relationship between the events reported on twitter and the incident reports available on curated sources such as 511.org that deals with San Francisco Bay area road traffic. We call the event extracted from twitter as corroborative to the event in 511.org if they are reporting exactly the same event. We call the extracted event to be complementary if it provides additional information about 511.org event, e.g., extracted event may be about traffic jam that further adds to the construction event given on 511.org. The extracted event (complementary or corroborative) is called timely if it precedes the event reported on 511.org. This can help explain some observations reported on conventional sources. For example, we extracted traffic event from a textual stream and a baseball game observation as reported from 511.org, using spatio-temporal context. Traffic information is complementary to the baseball game. If we extract baseball game event from textual stream, then the event will be corroborative (one supporting the other). If we extract any of the two events (traffic or baseball game in this example) before the incident report from 511.org then the extracted event is timely. We use these characteristics to manually verify each extracted event. From this discussion, the role and the benefits of relating multimodal data (that includes both IoT and social) for creating a reliable and comprehensive picture of a situation should be clear.

Orthogonal to the issues of exploiting multimodal data, we can also create rich background knowledge by combining probabilistic models grounded in numeric sensor data and declarative domain knowledge provided by domain experts in symbolic form. In other words, probabilistic graphical models build on the structural representation obtained using declaratively specified, qualitative dependencies among traffic parameters and concepts, while parameters reflecting relative likelihoods and conditional probabilities are estimated from the data. We have presented our preliminary work on creating such hybrid models by gleaning probabilistic knowledge from traffic data and using declarative knowledge from ConceptNet ${ }^{3}$ [3]. Figure 5 depicts the three sources of knowledge from which we can build probabilistic models: (a) Domain experts, (b) Domain observations, and (c) Domain knowledge.

\subsection{Health Care}

With increasing sensors and devices for monitoring physiological observations $[12,8,5]$, IoT can be significant in advancing state-of-the-art in health care and wellbeing [11]. Figure 6 demonstrates quantitative physiological observations reported by sensors and qualitative observations reported by people.

In what follows, we discuss the use of IoT for monitoring people and their environment, and show further benefits that accrue out of bringing patients in the loop.

Traditionally, people monitor personal health by visiting doctor for scheduled checkups or when sick. Recently, smartphones outfitted with numerous sensors (including environmental and physiological) have been increasingly used to monitor and manage personal health and well-being, over and beyond the periodic, but relatively infrequent, meetings with doctor. For example, we expect the actual causes/triggers

\footnotetext{
${ }^{3}$ http://conceptnet5.media.mit.edu/
} 
Figure 5: Creating probabilistic models (for answering diagnostic queries) using declarative knowledge from domain experts and knowledge bases

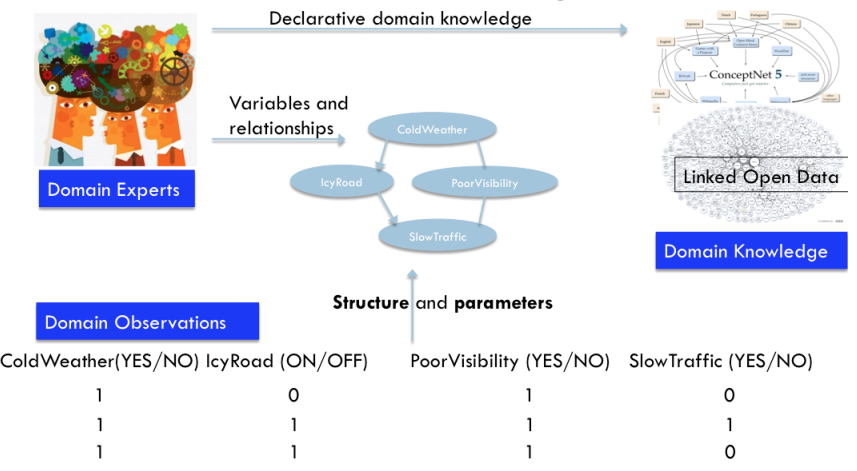
detailed monitoring of the environment and locations that an asthma patient visited, and the correspondence between symptoms and where/when they occurred, than was previously possible. The information that can be gleaned and abstracted from raw sensor data can be further compared against personal health data published by subscribers of Quantified Self movement, and obtained from social media. For another example, analysis of fine-grained sensor data from smartphone sensors such as accelerometer, compass, and microphone can be used to determine "visible/audible" symptoms (such as fall, unsteady motion, and slurrred speech) of Parkinson disease, and ultimately used to diagnose and assess its progression [4]. To realize these benefits, we need semantic perception to abstract fine-grained, machine accessible, heterogeneous sensor data to human comprehensible facts.

Deriving actionable insights requires multimodal data integration and abstraction of heterogeneous data, medical knowledge about symptoms and disorders, and the use of perception cycle embodying abductive reasoning for diagnosis and remedial action [7]. Measurements from sensors (such as thermometer, blood pressure gauge) can be abstracted to determine "objective" symptoms while "subjective" symptoms (such as are they feeling giddy or depressed) can be obtained only by interviewing or examining the patient. In general, if the observed symptoms are insufficient for actionable diagnosis, it is necessary to seek additional contextually relevant information for disambiguation, to arrive at a diagnosis, and from it, a remedial course of action. To realize these benefits, we need semantic perception that iteratively uses deductive reasoning for disambiguation and abductive reasoning for explanation generation, to arrive at the final diagnosis. Figure 7 illustrates how perception cycle can be used to narrow down the five possible explanations (that is, panic disorder, hypoglycemia, hyperthyroidism, heart attack, and septic shock) of the two observed "objective" symptoms (that is, abnormal heart rate and clammy skin), by systematically seeking additional "subjective" symptoms (through appropriate questioning in this case), to obtain the diagnosis, and eventually, prescription medications and alerts.
Figure 6: (a) Corroborative: wheezing reports corroborates exhaled Nitric Oxide (b) Complementary: reduced activity is complementary information to exhaled Nitric Oxide
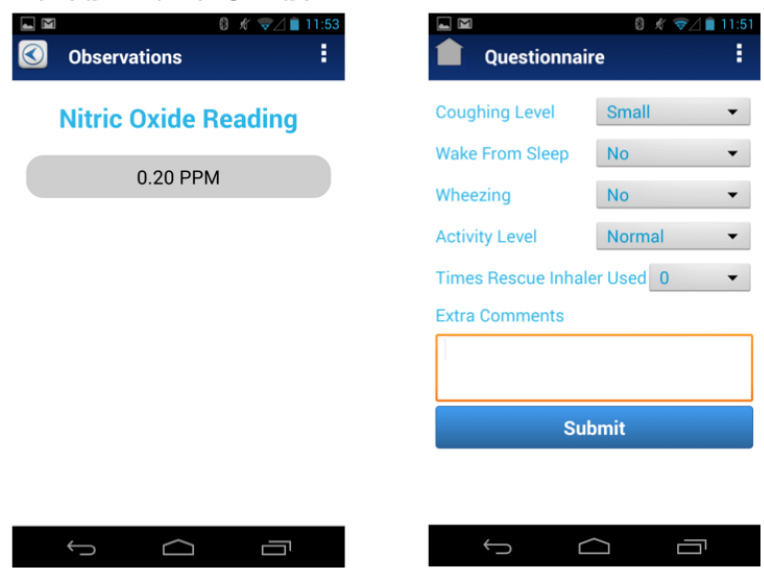

Figure 7: Perception cycle to seek additional symptoms in order to determine the diagnosis

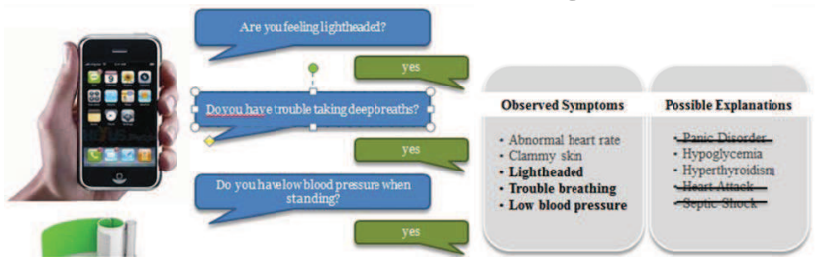

\section{CONCLUSIONS AND FUTURE DIREC- TION}

Observations from sensors and people are often complementary and can be combined for enhanced situational awareness. Interpretation of observations depends on the prior knowledge of the domain. Since each person possesses a personal prior knowledge (independent of others), variations in interpretation of observations is expected. With the proliferation of Physical-Cyber-Social systems, a systematic way of combining observations from IoT network with observations by people can be valuable for enhanced situational awareness.

There may be multiple explanations reported by people for the same sensor observations in many real-world situations. Finding the most likely explanation is an interesting line of research. For example, slow moving traffic may co-exist with accident or music/sporting event reported on textual stream. Finding the precise cause of slow moving traffic can be challenging in such situations.

\section{ACKNOWLEDGMENTS}

This work is partially undertaken under the EU FP7 Citypulse project contract number: 609035. We thank NSF for sponsoring SoCS grant IIS-1111182 titled as 'Social Media Enhanced Organizational Sensemaking in Emergency Response', to partially enable this work.

\section{REFERENCES}


[1] P. Anantharam, P. Barnaghi, K. Thirunarayan, and A. Sheth. Extracting city traffic events from social streams. 2014.

[2] P. Anantharam, K. Thirunarayan, and A. Sheth. Topical anomaly detection from twitter stream. In Proceedings of the 3rd Annual ACM Web Science Conference, pages 11-14. ACM, 2012.

[3] P. Anantharam, K. Thirunarayan, and A. Sheth. Traffic analytics using probabilistic graphical models enhanced with knowledge bases. 2nd International Workshop on Analytics for Cyber-Physical Systems (ACS-2013), 2013.

[4] P. Anantharam, K. Thirunarayan, V. Taslimi, and A. Sheth. Predicting parkinson's disease progression with smartphone data. In Technical Report, Submitted to the Parkinson's disease challenge sponsored by The Michael J. Fox Foundation for Parkinson's Research, March 2013.

[5] M. Chan, E. Campo, D. Estève, and J.-Y. Fourniols. Smart homescurrent features and future perspectives. Maturitas, 64(2):90-97, 2009.

[6] P. Cousin. Internt of things success stories, series 1. http:

//www.smart-action.eu/fileadmin/smart-action/
publications/IoT_Success_Stories_1.pdf, June 2014.

[7] C. A. Henson. A Semantics-based Approach to Machine Perception. PhD thesis, Wright State University, 2013.

[8] J. S. Karlsson, U. Wiklund, L. Berglin, M. Östlund, M. Karlsson, T. Bäcklund, K. Lindecrantz, and L. Sandsjö. Wireless monitoring of heart rate and electromyographic signals using a smart t-shirt. In Proceedings of International Workshop on Wearable Micro and Nanosystems for Personalised Health, 2008.

[9] K. Thirunarayan, P. Anantharam, C. Henson, and A. Sheth. Comparative trust management with applications: Bayesian approaches emphasis. Future Generation Computer Systems, 31:182-199, 2014.

[10] K. Thirunarayan and A. Sheth. Semantics-empowered big data processing with applications. 2014.

[11] E. J. Topol. The creative destruction of medicine: How the digital revolution will create better health care. Basic Books, 2012.

[12] S. Vaddiraju, I. Tomazos, D. J. Burgess, F. C. Jain, and F. Papadimitrakopoulos. Emerging synergy between nanotechnology and implantable biosensors: a review. Biosensors and Bioelectronics, 25(7):1553-1565, 2010. 